\title{
Определение параметров латерального и нестационарного шнурового токов в цилиндрическом халькогенидном стеклообразном полупроводнике
}

\author{
(С) Н.В. Совтус, К.Д. Мынбаев \\ Физико-технический институт им. А.Ф. Иоффре Российской академии наук, \\ 194021 Санкт-Петербург, Россия \\ E-mail: spnick93@mail.ru
}

Поступила в Редакцию 29 мая 2019 г.

В окончательной редакции 8 июля 2019 г.

Принята к публикации 10 июля 2019 г.

Для полупроводниковой системы $\mathrm{Ge}-\mathrm{Sb}-\mathrm{Te}$ цилиндрической конфигурации приближенно решено уравнение теплопроводности, описывающее токовый шнур в полупроводнике. Показано, что при бесконечно больших временах ток в шнуре пропорционален квадрату максимальной температуры в центре шнура и обратно пропорционален величине приложенного электрического поля. Оценен масштаб латерального тока, протекающего перпендикулярно току основного шнура, и установлено, что латеральный ток мал по сравнению с током, протекающим в шнуре, так что возникновение латеральных шнуров, растущих из основного шнура, мало вероятно.

Ключевые слова: халькогенидные стеклообразные полупроводники, эффект памяти, шнурование тока, латеральный ток.

DOI: $10.21883 /$ FTP.2019.12.48624.9176

\section{1. Введение}

Халькогенидные стеклообразные полупроводники (ХСП) исследуются с конца 50-х годов [1], и многие их свойства уже хорошо изучены. Особый интерес представляет эффект переключения в ХСП, при котором происходит управляемый с помощью напряжения обратимый токовый пробой и в определенной области полупроводника значительно возрастает производная от плотности протекающего тока по электрическому полю, называемая токовой дифференциальной проводимостью. Это явление называется шнурованием тока и означает концентрацию протекающего тока в данной координатной области. Шнурование тока интенсивно исследовалось с момента экспериментального обнаружения обратимого пробоя Овшинским в 1967 г. [2]. Был проведен ряд работ и по моделированию шнурования, но такие расчеты в основном либо проводились численно [3], либо для декартовой системы координат [4], либо не относились к ХСП [5]. В работе [3] исследовался случай исключительно теплового пробоя в ХСП. С 70-х годов началось исследование электротеплового пробоя, когда проводимость зависит не только от температуры, но и от электрического поля [6].

Появление токового шнура при эффекте переключения неразрывно связано с эффектом памяти на основе фазового состояния (Phase Change Memory, PCM), при котором в полупроводнике сохраняется то состояние, в которое он перешел под воздействием внешнего напряжения [7]. Эта способность ХСП позволяет использовать их в качестве носителей информации с большим (до $10^{8}$ ) количеством циклов перезаписи [8-11]. Знание как мож- но большего количества характеристик шнурового тока полезно при изготовлении как устройств с фазовой памятью, так и тиристоров, так как неоднородное распределение тока может повлечь за собой разрушение системы.

Цель данной работы заключалась в доказательстве существования устойчивого токового шнура, не способного распадаться во времени (в том числе и за счет диффузии). Авторами статьи было получено приближенное наглядное и максимально простое аналитическое описание поведения токового шнура в ХСП, учитывающее зависимость параметров последнего от времени и координат. На основании полученных результатов была выполнена оценка масштаба латерального тока, возникающего в полупроводнике из-за разницы температур в различных точках при образовании токовых неоднородностей. Было показано, что латеральные токи не приводят к разрушению токового шнура из-за своей малой величины по сравнению с током шнура.

\section{2. Аналитическое решение}

Рассмотрим образование токового шнура в цилиндрической полупроводниковой пластине с теплоотводом, осуществляющимся с торцов пластины по закону Ньютона. По умолчанию такие величины, как проводимость и энергия ее активации, возьмем для полупроводников типа GST ( $\mathrm{Ge}-\mathrm{Sb}-\mathrm{Te})$.

К торцам пластинки приложим постоянное напряжение. Проводимость $\sigma$ возьмем путем объединения 
проводимостей, используемых в [12,13]:

$$
\sigma=\sigma_{0} \exp \left(-\frac{\Delta E}{k T}+\frac{F}{F_{0}}\right)
$$

где $\Delta E$ - энергия активации проводимости, $T-$ температура, $k$ - постоянная Больцмана, $F$ - электрическое поле, $F_{0}$ - характеристический параметр.

Подобная зависимость для проводимости является самой простой из всех, учитывающих электротепловой пробой. На сегодняшний день предложены более совершенные модели, учитывающие влияние электрического поля, например модель $U$-центров [14], однако они весьма сложны для рассмотрения.

Гельмонт и Цэндин в [15] решили стационарное уравнение теплопроводности, описывающее распределение тока:

$$
t^{\prime \prime}(x)+\frac{t^{\prime}(x)}{x}-\left(t-t_{0}\right)+\beta \exp \left(-\frac{1}{t}\right)=0,
$$

где $t=\frac{k T}{\Delta E}-$ безразмерная температура, $x=\frac{r}{r_{0}}-$ безразмерная координата;

$$
\beta=\frac{F^{2}}{F_{s}^{2}} \exp \left(\frac{F}{F_{0}}\right), \quad F_{s}^{2}=\frac{2 \lambda \Delta E}{\sigma_{0} L k}, \quad F_{0} \approx 10^{6} \mathrm{~B} / \mathrm{M},
$$

$L-$ толщина пластинки, $\lambda-$ коэффициент теплоотвода.

В настоящей работе уравнение (2) было преобразовано в зависящее от времени $u$ :

$$
t^{\prime \prime}(x)+\frac{t^{\prime}(x)}{x}-\left(t-t_{0}\right)+\beta \exp \left(-\frac{1}{t}\right)=\frac{\partial t}{\partial u} .
$$

Для приближенного решения в аналитическом виде первое и второе слагаемые приравняли друг другу, чтобы получить в нуле ограниченное решение. Тогда нуль представляет собой точку экстремума. Слагаемое с экспонентой разложили вблизи максимальной температуры $t_{\max }$, допустимой в центре. Она выбрана не зависящей от времени. Окончательный вид и граничные условия:

$$
2 \frac{t^{\prime}(x)}{x}+a t+b=\frac{\partial t}{\partial u}
$$

$\frac{\partial t}{\partial x}(0, u)=0 ; \quad t(x, \infty)=t_{0}+\left(t_{\max }-t_{0}\right) \exp \left(-\frac{a x^{2}}{4}\right)$.

Решением уравнения (4) является выражение

$$
\begin{aligned}
t(x, u)= & -\frac{b}{a}+C_{1} \exp \left(-\frac{a x^{2}}{4}\right) \\
& -C_{1} \exp \left(-\frac{a x^{2}}{2}-a u\right)+C_{2} \exp (a u), \\
b= & \beta \exp \left(-\frac{1}{t_{\max }}\right)\left(1-\frac{1}{t_{\max }}\right)+t_{0} \\
a & =\frac{\beta}{t_{\max }^{2}} \exp \left(-\frac{1}{t_{\max }}\right)-1 ; \quad u=\frac{c \hat{t}}{r_{0}^{2}}
\end{aligned}
$$

где $\hat{t}-$ время, $r_{0}=1.4 \cdot 10^{-6} \mathrm{M}, c-$ температуропроводность $\left(c=2 \cdot 10^{-6} \mathrm{M}^{2} / \mathrm{c}\right)$.
Данное уравнение имеет три типа решений: однородное, неоднородное и решение с неограниченным нагревом пластинки. Последнее соответствует разрушению материала и не представляет интереса. Однородное решение появляется при условиях $C_{2}=0, a=0$. Также из условия $C_{2}=0$ следует $t_{0}=-\frac{b}{a}$, и в данном случае $b=0$.

Тогда выполняется переход к уравнению

$$
t_{\max }^{2}-t_{\max }+t_{0}=0 .
$$

Приближенные корни

$$
\begin{aligned}
& t_{m 1} \approx t_{0}+t_{0}^{2}, \\
& t_{m 2} \approx 1-t_{0}-t_{0}^{2} .
\end{aligned}
$$

Уравнение описывается функцией Хевисайда

$$
t(x, u)=t_{0}+\left(t_{\max }-t_{0}\right) \theta(u) \theta(x) .
$$

При $a>0$ появляется устойчивое во времени неоднородное решение (шнур)

$$
\begin{gathered}
C_{2}=0, \quad a>0, \\
t(x, u)=-\frac{b}{a}+\left(t_{\max }+\frac{b}{a}\right) \exp \left(-\frac{a x^{2}}{4}\right) \\
-\left(t_{\max }+\frac{b}{a}\right) \exp \left(-\frac{a x^{2}}{2}-a u\right), \\
t_{0}=-\frac{b}{a}, \quad t_{\max }^{2}-t_{\max }+t_{0}=0 .
\end{gathered}
$$

Условие $t(x)=t_{0}$ в начальный момент времени выполняется лишь вблизи нуля и на бесконечности; при $a \gg 1$ оно выполняется с достаточной точностью. При $u=0$ температура отлична от $t_{0}$ (моментальное образование токового расслоения), при этом максимума температура достигает не в центре шнура, а по его периметру, где идет самое мощное тепловыделение, как и было указано в [15] для стационарного случая. Затем за несколько десятков пикосекунд происходит перераспределение температуры, и она достигает максимума в центре полупроводника.

В точке $x=0$ уравнение (8) принимает экспоненциальный вид, описанный в [16], а при бесконечном времени получается стационарное решение (4), при котором производная по времени равна нулю [15].

Корнями последнего квадратного уравнения являются числа, приближенные значения которых сравнимы с таковыми для корней уравнения (6):

$$
t_{m 1,2}=\frac{1 \pm \sqrt{1-4 t_{0}}}{2}
$$

Полученные согласно (8) зависимости приведены на рис. 1. Из него можно определить радиус токового шнура - области, где выделяется максимальное количество 


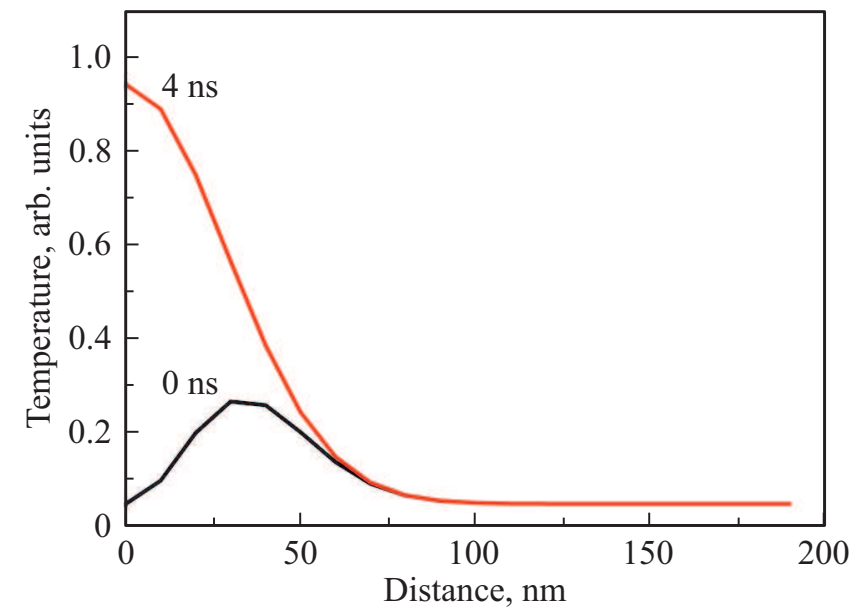

Рис. 1. Зависимость безразмерной температуры от расстояния до центра шнура, рассчитанная при $F=4 \cdot 10^{6} \mathrm{~B} / \mathrm{M}$, $F_{0}=6 \cdot 10^{6} \mathrm{~B} / \mathrm{M}, \alpha=100$.

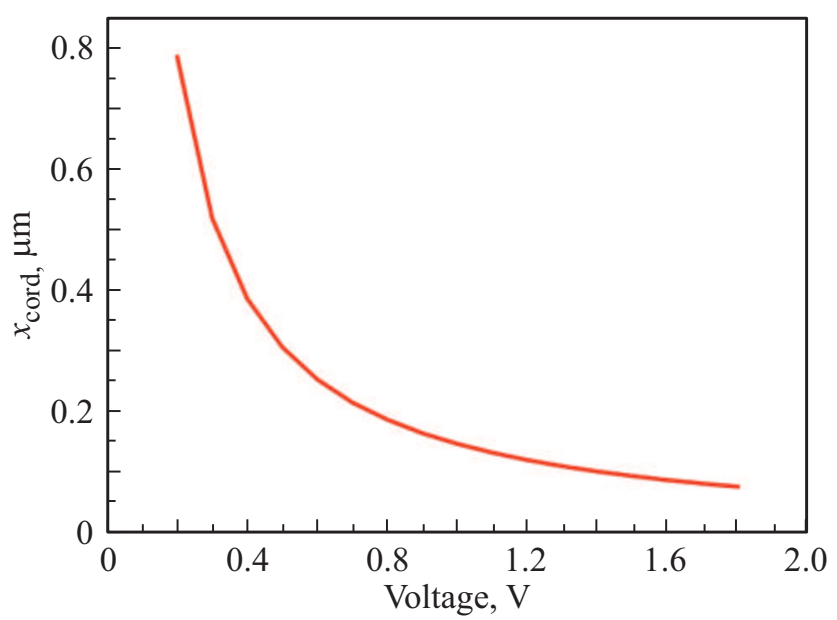

Рис. 2. Зависимость радиуса шнура от напряжения, рассчитанная для $t_{0} \approx 0.05, \Delta E=0.5$ эВ, $t_{\max }=0.94, L=10^{-6} \mathrm{M}, u=\infty$, $F_{0}=6 \cdot 10^{6} \mathrm{~B} / \mathrm{M}$.

тепла. За радиус шнура принята координата $\frac{\partial^{2} t}{\partial x^{2}}=0$. Это точка перегиба, в которой градиент модуля диффузионного потока из-за разницы температур достигает максимума.

Тогда

$$
\begin{aligned}
& t(x, u)=-\frac{b}{a}+\left(t_{\max }+\frac{b}{a}\right) \exp \left(-\frac{a x^{2}}{4}\right) \\
&-\left(t_{\max }+\frac{b}{a}\right) \exp \left(-\frac{a x^{2}}{2}-a u\right), \\
& x_{\text {cord }}(u=\infty)=\sqrt{\frac{2}{a}} ; \quad I=\langle\sigma\rangle \pi x_{\text {cord }}^{2} F \propto \frac{t_{\max }^{2}}{F} .
\end{aligned}
$$

При бесконечном времени эта зависимость для тока полностью совпадает с зависимостью от поля и максимальной температуры, полученной в $[15,17]$. Она показана на рис. 2. Здесь по оси абсцисс отложено напряжение, приложенное к противоположным сторонам диска, по оси ординат - радиус шнура $x_{\text {cord }}$ в микрометрах.

Рост температуры в центре шнура по формуле (10) происходит за сравнительно короткое время, это объясняется выбранной величиной коэффициента температуропроводности, соответствующей реальной величине для ХСП. Формулы для (6) частично совпадают с аналогичными формулами для определения максимальной температуры в центре, предложенными в [15]. Если посчитать, что $t_{\max }=t_{0}+t_{0}^{2}$, то из $a=1$ получится уравнение для определения порогового поля, приведенное в [15].

Оценить количество тепла, выделяемого при переключении, можно, используя закон Дюлонга-Пти и умножая теплоемкость на среднее отклонение температуры от комнатной:

$$
Q=3 N k\langle\Delta T\rangle=\frac{6 N \Delta E}{x_{\text {cord }}^{2}} \int_{0}^{x_{\text {cord }}^{2}}\left(t(x)-t_{0}\right) d x .
$$

Здесь $N$ - число атомов полупроводника, заключенных в объеме шнура. При $\Delta T=1000 \mathrm{~K}$ и $x_{\text {cord }}(\infty)=0.1$ получаем $Q \approx 10^{-11}$ Дж. Это значение очень хорошо согласуется с величиной $Q$, полученной экспериментально в [18], что подтверждает справедливость нашего подхода.

Если продифференцировать плотность шнурового тока по электрическому полю, то можно увидеть, что в некоторых случаях из формулы (10) следует отрицательная дифференциальная проводимость:

$$
\begin{gathered}
j=\sigma_{0} \exp \left(-\frac{\Delta E}{k T}+\frac{F}{F_{0}}\right) F \Rightarrow \frac{d j}{d F} \\
=\sigma_{0} \exp \left(-\frac{1}{t}=\frac{F}{F_{0}}\right)\left(\frac{t_{F}^{\prime}}{t^{2}}=\frac{F}{F_{0}}=1\right), \\
\frac{d a}{d F}=2 \frac{(a+1)}{F}+\frac{a+1}{F_{0}}>0 .
\end{gathered}
$$

Шнур тока существует при выполнении неравенства $t_{F}^{\prime}>-t^{2}\left(F / F_{0}+1\right)$. Это область положительной дифференциальной проводимости, так как согласно формуле (10) и результатам [15,17], при увеличении поля радиус токового шнура уменьшается и, следовательно, плотность тока должна возрасти.

\section{3. Латеральный ток}

Общий латеральный (т.е. протекающий перпендикулярно шнуру) ток вычисляется по формуле

$$
j_{\text {side }}=j_{n}=j_{\text {diff }}=\sigma_{n}\left(\frac{d \varphi}{d x}+\frac{k T}{e n} \frac{d n}{d x}\right) .
$$

Первое слагаемое в этом выражении отвечает за дрейфовый ток $j_{n}$, возникающий у заряженных частиц в электрическом поле, второе - за ток диффузии 


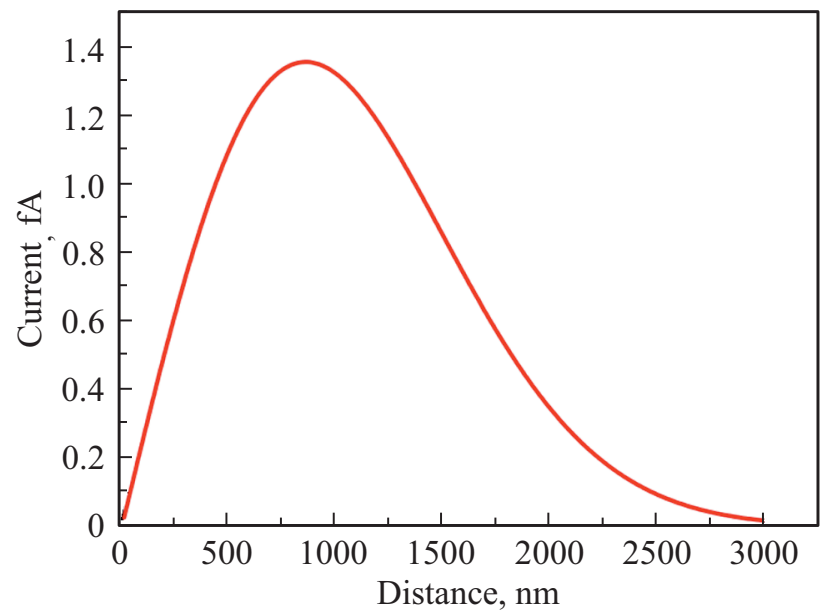

Рис. 3. Зависимость дрейфового тока от расстояния до центра шнура, рассчитанная для $\hat{t}=7 \mathrm{Hc}, U=2 \mathrm{~B}, t_{0}=0.05$, $F_{0}=6 \cdot 10^{6} \mathrm{~B} / \mathrm{M}$.

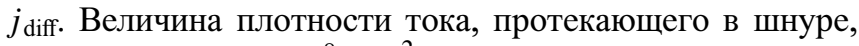
составляет $j_{\text {cord }} \sim 10^{8} \mathrm{~A} / \mathrm{M}^{2}[14]$.

Дрейф электронов происходит в поле $\varphi$, создаваемом за счет обусловленной градиентом температуры разности потенциалов вдоль радиальной составляющей, $k T=e \varphi$ :

$$
j_{n}=\sigma_{n} \frac{k \nabla T}{e}=\sigma_{n} \frac{\Delta E \nabla t}{e} .
$$

При $t \sim 0.1$ плотность дрейфового тока $j_{n} \sim 0.1 \mathrm{~A} / \mathrm{M}^{2}$. Таким образом, величина дрейфового тока оказывается на несколько порядков меньше тока, протекающего в шнуре. Зависимость дрейфового тока от координаты при заданном времени приведена на рис. 3

Для оценки второго слагаемого в скобках в выражении (13) было взято выражение для подвижности $\mu$ из работы [13]:

$$
\mu=\mu_{0} N_{c} / N_{t} \exp \left(-\frac{\Delta E}{k T}\right) .
$$

Здесь $\mu_{0}-$ подвижность заряда в разрешенной зоне, $N_{c}$ - эффективная плотность состояний в разрешенной зоне, $N_{t}$ - плотность локализованных состояний. Проводимость определяется известной формулой $\sigma=e n \mu$, Тогда с учетом выражений (1) и (13) получается, что концентрация носителей не зависит от температуры и координаты, и, следовательно, диффузионный ток отсутствует.

Однако если предположить, что $\mu \propto \exp (-m / t)$, где $m$ - рациональное число, то $n=n_{0} \exp (-p / t)$, $m+p=1$. Выражение для диффузионного тока тогда приобретает вид:

$$
j_{\text {diff }} \propto \frac{\sigma_{n}}{e} \operatorname{axp} \Delta E
$$

Максимальная величина диффузионного тока, описываемого выражением (16), не превышает $10^{4} \mathrm{~A} / \mathrm{M}^{2}$.
Отметим, что формула (16) действительна только для небольших значений $x$, т.е. вблизи шнура. Здесь $\sigma_{0}=5 \cdot 10^{3} \mathrm{OM} \cdot \mathrm{M}, \Delta E=0.5$ эВ, $a \sim 10^{2}, F \sim 10^{6} \mathrm{~B} / \mathrm{M}$.

Если сравнить выражения (16) и (13) и принять, что $\nabla t \propto a x t$, то можно прийти к неравенству $p \geq t$. Если оно выполняется, то диффузионный ток превышает дрейфовый, и наоборот. В любом случае латеральный ток оказывается на несколько порядков меньше шнурового тока, а значит, не может приводить к разрушению шнура.

Отметим, что у предложенного подхода есть и свои недостатки: в частности, решение было получено с учетом классической формы для электронной проводимости и не учитывало ее более сложную зависимость от поля. Эти вопросы планируется подробно рассмотреть в последующих работах.

\section{4. Заключение}

В работе была построена максимально простая аналитическая модель шнурования тока в халькогенидном стеклообразном полупроводнике с учетом зависимости от координаты и времени. Эта модель была использована для оценки масштаба токов, перпендикулярных току шнура и возникающих из-за неравномерного температурного разогрева полупроводника. Показано, что ток шнура способен удерживаться в течение долгого времени, не разрушаясь. Формула, описывающая шнур, имеет физический смысл и согласуется с данными, полученными ранее, но справедлива только для сравнительно небольших расстояний, приблизительно равных радиусу токового шнура. Показано, что латеральный ток не влияет на параметры шнура, так как его максимальная величина на несколько порядков ниже величины тока в шнуре. Таким образом, возникновение боковых каналов токового шнура, грозящих материалу повреждениями, крайне мало вероятно.

\section{Конфликт интересов}

Авторы заявляют, что у них нет конфликта интересов.

\section{Список литературы}

[1] B.T. Kolomiets. Phys. Status Solidi B, 7, 359 (1964).

[2] S. Ovshinsky. Phys. Rev. Lett., 21, 1450 (1968).

[3] N. Croitoru, C. Popescu. Rev. Rom. Phys., 16, 129 (1971).

[4] D. Thomas, J. Male. J. Non-Cryst. Sol., 8-10, 522 (1972).

[5] И.В. Печенежский, С.И. Дорожкин. Письма ЖЭТФ, 88 (2), 137 (2008).

[6] N. Bogoslovskiy, K. Tsendin. Solid-State Electron., 129, 10 (2017).

[7] M. Nardone, M. Simon, I.V. Karpov, V.G. Karpov. J. Appl. Phys., 112, 071101 (2012).

[8] H.S.P. Wong, S. Raoux, S.B. Kim, J. Liang, J.P. Reifenberg, B. Rajendran, M. Asheghi, K.E. Goodson. Proc. IEEE, 98, 2201 (2010). 
[9] G.W. Burr, M.J. Brightsky, A. Sebastian, H.Y. Cheng, J.Y. Wu, S. Kim, N.E. Sosa, N. Papandreou, H.L. Lung, H. Pozidis, E. Eleftheriou, C.H. Lam. IEEE J. Select. Top. Circ. Syst., 6, 146 (2016).

[10] W. Zhang, R. Mazzarello, M. Wuttig, E. Ma. Nature Rev. Mater., 4, 150 (2019).

[11] P. Yeoh, Y. Ma, D.A. Cullen, J.A. Bain, M. Skowronski. Appl. Phys. Lett., 114, 163507 (2019).

[12] А.И. Исаев, С.И. Мехтиева, С.Н. Гарибова, В.З. Зейналов. ФТП, 46, 1138 (2012).

[13] Э.А. Лебедев, С.А. Козюхин, Н.Н. Константинова, Л.П. Казакова. ФТП, 43, 1383 (2009).

[14] К.Д. Цэндин, Н.А. Богословский. ФТП, 46, 577 (2012).

[15] Б.Л. Гельмонт, К.Д. Цэндин. Электронные явления в некристаллических полупроводниках (Л., Наука, 1976) c. 177.

[16] A.H.M. Shousha. J. Appl. Phys., 42, 5131 (1971).

[17] Б.Л. Гельмонт, К.Д. Цэндин. ФТП, 10, 1119 (1976).

[18] А.М. Попов, С.М. Сальников, Ю.В. Ануфриев. ФТП, 49, 509 (2015).

Редактор Г.А. Оганесян

\title{
Parameters of lateral and unsteady cord currents in a cylindrical chalcogenide glassy semiconductor
}

\section{N.V. Sovtus, K.D. Mynbaev}

loffe Institute,

194021 St. Petersburg, Russia

\begin{abstract}
For the $\mathrm{Ge}-\mathrm{Sb}-\mathrm{Te}$ system, the heat equation describing the current cord in the cylindrical semiconductor plate is approximately solved. It is shown that for infinitely large times the current in the cord is proportional to the square of the maximum temperature in the center of the cord and inversely proportional to the applied electric field. The scale of the lateral current flowing perpendicular to the cord is estimated, and it is established that the lateral current is small as compared to the current flowing in the cord, so the occurrence of lateral cords growing from the main cord is unlikely.
\end{abstract}

